\title{
Neutropenia and T-Wave Inversion as Toxin-Mediated Complications of a Streptococcal Infection
}

\author{
Cristina Popescu1,2, Anca Leuştean ${ }^{1,2}$, Alina Elena Orfanu1,2*, Codruţa Georgiana Carp², \\ Victoria Aramă 1,2 \\ 1 "Carol Davila" University of Medicine and Pharmacy, Bucharest, Romania \\ 2 "Prof. Dr. Matei Balş" National Institute for Infectious Diseases, Bucharest, Romania
}

\begin{abstract}
Introduction: Streptococcal infection can be responsible for multiple complications, such as toxic, septic or allergic disorders. Toxin-mediated complications (TMC) can appear during the acute phase of disease and can involve any organ, causing carditis, arthritis, nephritis, hepatitis etc.

Case presentation: The case of a young woman without a history of recurrent streptococcal tonsillitis, admitted to "Matei Balş" National Institute for Infectious Diseases, Bucharest, Romania, presenting with fever, sore throat and exudative tonsillitis, is detailed. The initial test for Streptococcus pyogenes was negative. The patient had leukopenia with severe neutropenia, high values of inflammatory biomarkers and electrocardiographic (ECG) changes with inverted T waves in leads V1-4 and flattened T waves in V5-6. There were no other cardiac signs or symptoms. The patient received cefuroxime for two days. On admission, the patient was prescribed Penicillin $\mathrm{G}$ and dexamethasone which resulted in a rapid recovery. The leucocytes count returned to normal as did the ECG abnormalities. At the time of discharge, the antistreptolysin 0 titre was high.
\end{abstract}

Conclusions: The case highlights the toxin-mediated complications (TMC) of streptococcal infection which occur from the outset of the disease.

Keywords: tonsillitis, streptococcal infection, leukopenia, toxic myocarditis

Received: 10 August 2017 / Accepted: 23 October 2017

\section{INTRODUCTION}

The most frequent aetiology of acute pharyngitis is represented by respiratory viruses that usually cause symptoms like conjunctival hyperaemia, rhinorrhoea, sore throat, laryngitis or cough. Exudative pharyngitis is not common in viral infections, except for the Epstein-Barr virus (EBV) infection. Streptococcus pyogenes is the most common bacterial agent involved in exudative pharyngitis, being responsible for $5 \%$-30\% of all cases of acute tonsillitis, especially in children [1]. Streptococcal pharyngitis can be associated with multiple complications such as toxin-mediated (TMC), septic or allergic complications. TMC due to streptococcal infections are rarely reported in the literature, but when reported, any organ can be affected, causing arthritis, nephritis, hepatitis, haematological or cardiac disorders or even toxic shock [2, 3]. Several cases of haematological disorders [4] or ECG abnormalities [5] due to streptococcal infection have been reported in the medical literature, though none can be traced regarding the association between $\mathrm{T}$-wave inversion and neutropenia.

\section{CASE PRESENTATION}

A 31-year-old woman, without a medical history of streptococcal tonsillitis, was admitted, presenting with fever, headache, sore throat, tachycardia and fatigue. The onset of the disease had occurred three days before the admission. The patient had, prior to admission, cefuroxime $500 \mathrm{mg}$ twice a day without improvement.

On admission, the patient's temperature was recorded as $39.5^{\circ} \mathrm{C}$. Clinical exam showed enlarged tonsils 
with extended white spots on both tonsils and submandibular lymphadenopathy. ECG, complete blood count (CBC), markers of inflammation, biochemistry (renal, liver and metabolic tests), urinalysis, a throat swab and a rapid test for streptococcal infection were carried out. The patient was hospitalized having presented with a leukocyte count of 1800 leukocyte/ $\mathrm{mm}^{3}$, a neutrophil count of 300 neutrophils $/ \mathrm{mm}^{3}$, inverted $\mathrm{T}$ waves in leads V1-4 (Figure 1) and negative "rapid strep test". After that, we completed the investigations as follows:

- transaminases, electrolytes, lactate dehydrogenase $(\mathrm{LDH})$, creatin kinase-MB (CK-MB) and troponin; all the mentioned tests were within normal values;

- antistreptolysin O test (ASLO) below 200 IU/ $\mathrm{ml}$;

- blood smear with leukopenia and neutropenia, without blast cells;

- inflammatory syndrome (C reactive protein 123 $\mathrm{mg} / \mathrm{L}$, fibrinogen $8.18 \mathrm{~g} / \mathrm{L}$ );

- procalcitonin level under $0.05 \mathrm{ng} / \mathrm{ml}$;

- viral capsid antigen (VCA) IgM for EBV - nega- tive, with positive IgG;

- thyroid tests within normal limits;

- transthoracic echocardiography without signs of pericarditis.

From the beginning, four possible scenarios were taken into consideration for the differential diagnosis: a streptococcal infection, a viral infection, a bacterial tonsillitis of other aetiology and a haematological disorder.

\section{Streptococcal infection}

A streptococcal pharyngitis is the most frequent aetiology of exudative tonsillitis. Moreover, the TMC during a streptococcal infection can include cardiac or haematological toxicity. The severe inflammatory syndrome on admission and the increasing value of ASLO at discharge sustain this supposition. The rapid strep test and the cultures were negative, but can be explained by the fact that the patient received antimicrobial therapy active on Streptococcus pyogenes prior to admission.

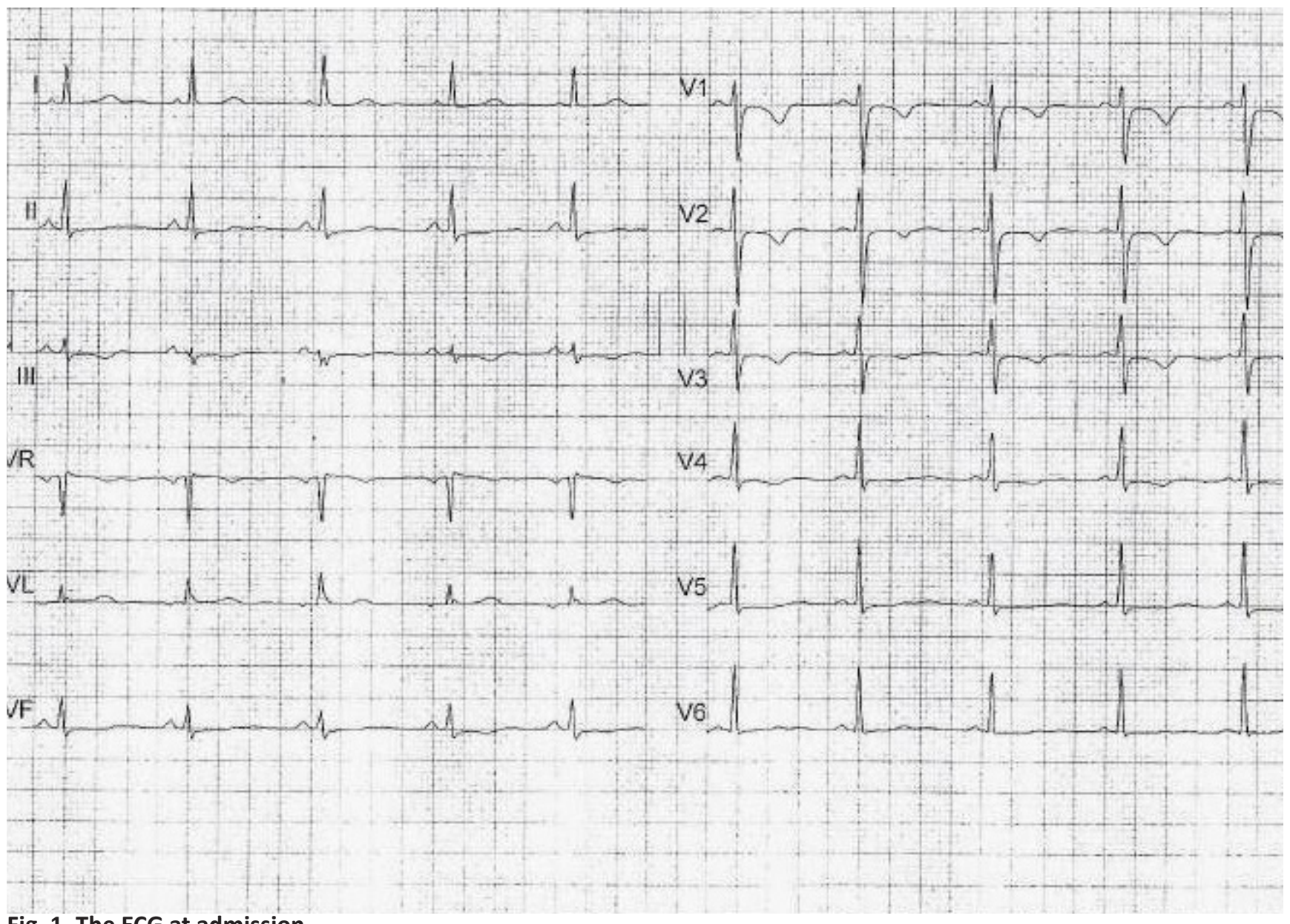

Fig. 1. The ECG at admission 


\section{A viral infection}

A viral infection, especially an infectious mononucleosis, the most common cause of viral exudative tonsillitis, was excluded because the patient was previously infected with EBV (positive anti VCA IgG for EBV). Furthermore, we did not find generalized lymphadenopathy, hepatosplenomegaly, increased transaminases which are suggestive elements for EBV primary infection.

\section{Bacterial tonsillitis of other aetiology}

Bacterial tonsillitis of other aetiology, especially a staphylococcal infection; the TMC of staphylococcal infection involves especially skin disorders and it is unusual to associate leukopenia and ECG changes. Diphtheria has been eradicated in Romania and therefore was ruled out. Moreover, the symptomatology was not suggestive for this aetiology.

\section{A haematological disorder}

This was excluded after haematological evaluation, including a blood smear. The haematologist did not con- sider necessary a bone aspiration or biopsy.

The conclusion was that the most probable aetiology was Streptococcus pyogenes. The patient received intravenous Penicillin G ( 1 million units every 6 hours) in association with oral slow-releasing clarithromycin (500 mg/day) and dexamethasone (4mg twice a day).

The clinical outcome was good, but after 24 hours the leucocyte count decreased to $1100 / \mathrm{mm}^{3}$ with 200 neutrophils $/ \mathrm{mm}^{3}$. After 48 hours, the leucocyte count was $4600 / \mathrm{mm}^{3}$ with normal neutrophil count. The ECG was repeated after 48 hours and it showed normal sinus rhythm and cardiac axis (+30 degrees), but with bradycardia (55/minute) and the persistence of T-wave inversion in leads V1-3, with almost normal $\mathrm{T}$ waves in V5-6 (Figure 2). The biological markers for myocardial necrosis (troponin, LDH, CK-MB) remained normal after 48 hours. We stopped clarithromycin after 2 days because a staphylococcal infection was excluded. In day 3 of admission, the clinical exam showed the decrease of tonsils volume without any exudate at this level. The submandibular lymphadenopathy has also decreased in volume. After 96 hours, the ECG revealed T waves

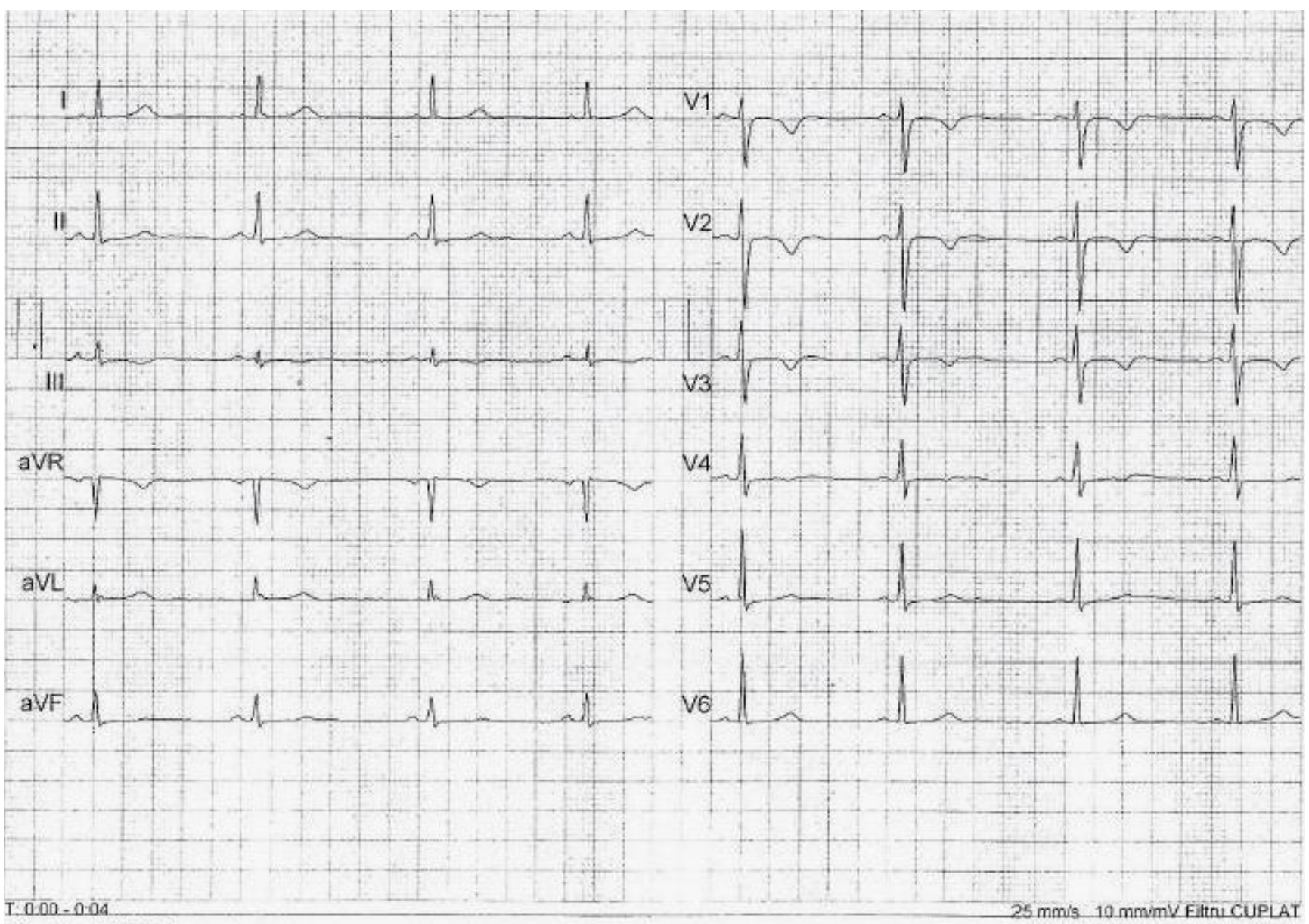

Fig. 2. The ECG after 48 hours 
with favourable outcome, with inverted $\mathrm{T}$ waves only in leads V1-2, prolonged QT interval and persistent bradycardia (Figure 3 ). The cardiologist did not consider necessary to perform any supplementary evaluation. The leucocyte count increased to $12000 / \mathrm{mm}^{3}$ and we stopped the dexamethasone. We discharged the patient after 6 days, with normal leucocyte count and inflammatory biomarkers. ASLO titre increased 3 fold.

Oral penicillin 1 million units every 6 hours for another 4 days was prescribed.

One week after discharge, the ECG showed normal heart rate, inverted T waves only in V1-2 and a normal QT interval.

\section{DISCUSSIONS}

In this case report, we describe the TMC of streptococcal tonsillitis such as T-wave inversion syndrome and severe leukopenia and neutropenia in a patient without any history of recurrent streptococcal infection and also without cardiac or haematological antecedents. Streptococcus pyogenes is the most common aetiology of exudative pharyngitis and in more rare cases, some viruses or other bacteria can be involved. This is the main reason why swabbing the throat should be performed from the beginning, before starting the antimicrobial therapy, to identify the pathogen agent.

The pathogenesis of streptococcal infections involves multiple toxins or virulence factors such as exotoxins A, B, C, F, streptococcal superantigens released into the circulation in the acute phase of infection. A high number of T-lymphocytes is activated, which is responsible for releasing inflammatory cytokines into the blood, causing several organic dysfunctions [6].

Myocarditis related to streptococcal infection is usually associated with rheumatic fever, 7-10 days after an acute episode of tonsillitis. The mechanism is probably a cross-reaction between streptococcal $\mathrm{M}$ proteins and cardiac myosin [7]. The first case of myocarditis unrelated to rheumatic fever was reported in 1947 [8]. Since then, several other cases, usually describing young patients without a history of cardiac pathologies, have been published, with previously unspecified clinical

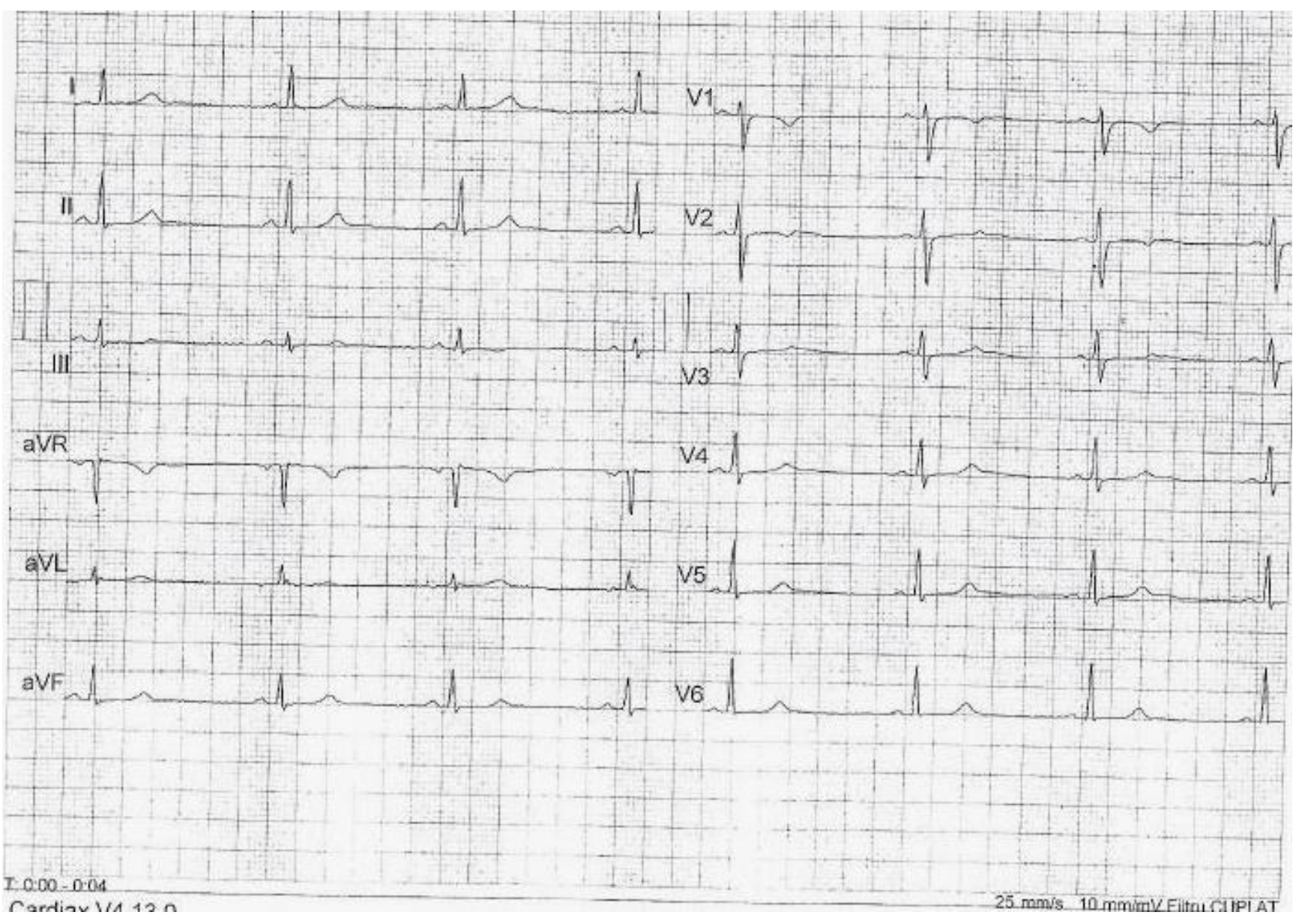

Fig. 3. The ECG after 96 hours 
signs or symptoms and ECG changes in the acute phase of a streptococcal tonsillitis $[9,10]$.

The most frequent ECG finding in streptococcal myocarditis is a diffuse T-wave inversion [11]. It is mandatory to differentiate between a normal variant, which is more frequent in young women than in men [12], an acute coronary syndrome and a transient T-wave inversion related to a streptococcal infection. Because the patient had previously normal ECG and cardiac enzymes and the ECG improved under antimicrobial therapy during hospitalization, we concluded that the $\mathrm{T}$-wave inversion was infection-related.

Concerning the haematological disorders, streptococcal infections are associated with leucocytosis with a high number of neutrophils, due to the intense activation of the immune system. In rare cases, leukopenia can occur and this is thought to be a toxin-mediated complication during acute tonsillitis. Other haematological disorders have also been associated with acute streptococcal infection, such as haemolytic anemia or thrombocytopenia. The haemolytic anemia can appear as an effect of the haemolytic enzymes contained by the bacteria [4]. Regarding the thrombocytopenia, it can be caused by $\mathrm{M}$ proteins' property to stimulate platelets activation [13].

A low number of cases reporting either T-wave inversion or neutropenia as TMC of streptococcal infections have been reported in the literature [14]. Our case is probably the first one reported until present regarding this association in a patient without previous cardiac or haematological disorders.

The sudden onset and the rapid recovery following antimicrobial therapy to combat Streptococcus pyogenes, sustains a toxic pathogenesis hypothesis. If ECG disturbances in such situation are accompanied by suggestive symptomatology or increased cardiac enzymes, further investigations as cardiac MRI may be necessary [15]. Unfortunately, in the absence of symptoms, the TMC of streptococcal infections remain under-recognized and under-reported.

\section{CONCLUSIONS}

It is important to perform an ECG in order to early diagnose a myocarditis when confronted by a patient with exudative tonsillitis, even though the patient does not have cardiac symptomatology. Further investigations are necessary if a myocarditis is suspected. Leukopenia with neutropenia, in a patient with exudative tonsillitis and an inflammatory syndrome, may also suggest a TMC related to a streptococcal infection.

\section{AUTHORS' CONTRIBUTIONS STATEMENT}

The authors have equally contributed to this paper. All the authors treated the patient, contributed to the writing of the draft and approved the final manuscript.

\section{CONFLICTS OF INTEREST}

The authors declare no conflicts of interest.

\section{ACKNOWLEDGEMENTS}

Written informed consent was obtained from the patient to publish the clinical details and images in this article. We thank Bogdana Manu, our cardiologist.

This work is part of "Carol Davila" doctoral programme.

\section{REFERENCES}

1. Wessels MR. Streptococcal pharyngitis. N Engl J Med. 2011;364:648-655.

2. Chuang YY, Huang YC, Lin TY. Toxic shock syndrome in children: Epidemiology, pathogenesis, and management. Paediatr Drugs. 2005;7:11-25.

3. Stevens DL. Streptococcal Toxic-Shock Syndrome: Spectrum of Disease, Pathogenesis, and New Concepts in Treatment. Emerg Infect Dis. 1995;1(3):69-78.

4. Tilanus $A M R$, de Geus HRH, Rijnders BJA, Dwarkasing RS, van der Hoven B, Bakker J. Severe group A streptococcal toxic shock syndrome presenting as primary peritonitis: a case report and brief review of the literature. Int J Infect Dis. 2010;14(3):20812.

5. Boruah P, Shetty S, Kumar SS. Acute streptococcal myocarditis presenting as acute ST-elevation myocardial infarction. J Invasive Cardiol. 2010;22(10):E189-91.

6. Cunningham MW. Pathogenesis of Group A Streptococcal Infections. Clin Microbiol Rev. 2000;13(3):470-511.

7. Burova LA, Nagornev VA, Pigarevsky PV, et al. Induction of myocarditis in rabbits injected with group A streptococci. Indian J Med Res. 2004;119:183-5.

8. Gore I, Saphir O. Myocarditis associated with acute nasopharyngitis and acute tonsillitis. Am Heart J. 1947;34(6):831-51.

9. Talmon Y, Gilbey P, Fridman N, Wishniak A, Roguin N. Acute myopericarditis complicating acute tonsillitis: beware the young male patient with tonsillitis complaining of chest pain. 
Available online at: www.jccm.ro

Ann Otol Rhinol Laryngol. 2008;117(4):295-7.

10. Upadhyay GA, Gainor JF, Stamm LM, Weinberg AN, Dec GW, Ruskin JN. Acute nonrheumatic streptococcal myocarditis: STEMI mimic in young adults. Am J Med. 2012; 125(12):1230-3.

11. Feldman AM, McNamara D. Myocarditis. N Engl J Med. 2000;343(19):1388-98.

12. Said SAM, Bloo R, de Nooijer R, Slootweg A. Cardiac and noncardiac causes of T-wave inversion in the precordial leads in adult subjects: A Dutch case series and review of the literature. World J Cardiol. 2015;7(2):86-100.

13. Shannon O, Hertzén E, Norrby-Teglund A, Vons C, Jacobs
The Journal of Critical Care Medicine 2017;3(4) • 171

F, Gordji-Therani H. Severe streptococcal infection is associated with $\mathrm{M}$ protein-induced platelet activation and thrombus formation. Mol Microbiol. 2007;65:1147-57

14. Kanetake K, Hayashi M, Hino A, et al. Primary peritonitis associated with streptococcal toxic shock-like syndrome: report of a case. Surg Today. 2004;34(12):1053-56

15. Mavrogeni S, Bratis K, Kitsiou A, Kolovou G. Streptococcal tonsillitis and acute streptococcal myocarditis: an unusual combination assessed by cardiac magnetic resonance imaging and endomyocardial biopsy. Ann Otol Rhinol Laryngol. 2012;121(9):604-8. 